\title{
Novel Method for Laparoscopic Common Bile Duct Exploration Performed Using a Disposable Bronchoscope (Ambu ${ }^{\circledR}$ aScope TM).
}

\author{
Yousif Mahmood Aawsaj \\ Specialist Registrar General Surgery \\ University Hospital of North Durham, \\ County of Durham and Darlington NHS foundation \\ Trust, UK \\ yousifaawsaj@doctors.org.uk
}

\author{
Ibrahim K Ibrahim \\ Clinical fellow in surgery \\ Freeman Hospital/ Newcastle NHS \\ foundation trust, UK \\ ibracadabra87@outlook.com
}

\author{
Andrew Gilliam \\ Consultant Upper GI surgeon \\ University hospital of north Durham/ \\ County of Durham and Darlington NHS foundation \\ Trust, UK \\ andrew.gilliam@nhs.net
}

\begin{abstract}
Laparoscopic common bile duct (CBD) exploration can be performed following choledocotomy or via the trancystic approach. Laparoscopic CBD exploration is limited in some benign upper gastrointestinal units due to the cost of sterilization of the re-usable choledochoscope. We have recently published a case series confirming the safety and efficacy of the $5 \mathrm{~mm}$ re-usable bronchoscope for CBD exploration. This case series evaluates a single-use bronchochoscope (Ambu aScopeTM) for laparoscopic CBD exploration. Data were collected from electronic records of the patients from January 2015 until December 2016; all the cases had confirmation of CBD stones. Twenty-one patients had CBD exploration using the disposable bronchochoscope. There were 7 male and 14 female (median age 42). 8 procedures were performed as emergencies and 13 were performed electively. All the cases were done laparoscopically apart from one planned as an open procedure due to previous extensive open surgery. Twenty cases had their CBD cleared using disposable bronchoscope and one needed subsequent special test follow up. Choledocotomy was performed in 15 and Tran's cystic approach was performed in 6. No T-tube was used in the laparoscopic cases. 2 cases were performed as day case surgery. Median postoperative hospital stay was 2.5 days. In conclusion, the disposable bronchoscope is safe and effective for use in CBD exploration with results comparable to our previously published case series. It has guaranteed sterility and is cost effective compared to the re-usable bronchoscope especially when initial capital outlay, sterile processing and maintenance costs are considered.
\end{abstract}

Keywords: laparoscopic Common bile duct exploration, disposable bronchoscope (Ambu ${ }^{\circledR}$ aScope TM)

\section{INTRODUCTION}

There are two commonly adopted approaches to treat common bile duct (CBD) stones, the single stage procedure of laparoscopic cholecystectomy and CBD exploration or endoscopic retrograde cholangiopancreatography (ERCP) followed by laparoscopic cholecystectomy. Recently, a published meta-analysis has shown no difference in the outcome between laparoscopic CBD exploration and ERCP in terms of duct clearance, morbidity and mortality [1].
The current practice of CBD exploration involves the use of a re-usable sterilized choledocoscope introduced to the duct via either choledocotomy or the transcystic approach. However, many benign upper gastrointestinal units are struggling to manage the logistics and cost of sterilization. Over last two years, we have been using disposable bronchoscope for laparoscopic CBD exploration. There is one case report showed successful use of this kit for this procedure [2]. The feasibility of using disposable bronchoscope for laparoscopic CBD exploration has been presented in case series that we published from different institution [3].

The aim of this series to show, in line with our previous work, the safety and efficacy of using disposable bronchoscope for laparoscopic CBD exploration.

\section{METHOD}

The study period was from January 2015 to December 2016 when we began to use the Ambu ${ }^{\circledR}$ aScope 2 TM in our institution. All cases required a laparoscopic common bile duct exploration were included. Data was collected retrospectively from electronic record of patients and analyzed retrospectively. Patients were consented for using this equipment to explore common bile duct and the company manufactured disposable bronchoscope has given the permission to use it for common bile duct exploration.

\section{Equipment and procedure:}

The Ambu ${ }^{\circledR}$ aScope 2TM was used for common bile duct exploration. This is a sterile, single use flexible bronchoscope. It is currently used in bronchoscopy and in tracheal intubation which is available in two sizes (3.8 $\mathrm{mm}$ and $5 \mathrm{~mm})$. It is a one piece unit with a single dimensional flexible tip manipulated from a hand piece (150 degree flex in the $5 \mathrm{~mm}$ model and 130 degrees in the $3.8 \mathrm{~mm}$ model). We only used $5 \mathrm{~mm}$ bronchoscope in our series. There is a single instrument channel which has a diameter of $2.2 \mathrm{~mm}$. This allows the passage of standard endoscopic baskets. The light source comes from a series of LED lights on the tip of the instrument. The image is projected to a high resolution 6.5 " LCD screen with a resolution of $640 \times 480$ pixels. It can be connected to a larger monitor via a DPI port. There is a suction port on the hand piece for bronchoscopy which use for connection of water irrigation tubing. This necessitates a standard 3 way connector. 
Our standard laparoscopic CBD exploration was performed using four ports. All cases included for CBD exploration were eligible when $\mathrm{CBD}$ diameter more than $10 \mathrm{~mm}$. Choledoctomy was performed using choledocotome or disposable aspiration needle; transcystic approach was performed following cystic duct dilatation. Absorbable 3/0 suture was used to close the choledocotomy and in these cases two drains were used one in sub-hepatic and the other in sub-phrenic spaces. T-Tube was used only when there was concern of retained stones.

\section{RESULTS}

Twenty one patients had CBD exploration using the disposable bronchochoscope. There were 7 male and 14 female. Patients median age was 42 (range 15 to 81 years). 8 procedures were performed as emergencies and 13 were performed electively. All the cases were done laparoscopically apart from one planned as an open procedure due to previous extensive open surgery. There was no conversion in laparoscopic cases. Ninteen cases were performed using disposable bronchochoscope primarily and two cases had their CBD explored after unsuccessful ERCP.

Twenty cases had their CBD cleared using disposable bronchoscope and one needed subsequent ERCP due to impacted retained stone. Choledocotomy was performed in 15 and transcystic approach was performed in 6. No T-tube was used in the laparoscopic cases. 2 cases were performed as day case surgery and there were performed using transcystic approach. Median overall hospital stay was three days (range 1 to 9 days) Median post-operative hospital stay was 2.5 days (range 1 to 4 days). There was two patient had umbilical port site wound infection and one had non-specific chronic abdomen pain.

\section{DISCUSSION AND CONCLUSION}

The re-usable choledocoscope has been used conventionally for CBD exploration. The cost associated with using the standard re-usable choledocoscope has limited the number of CBD exploration by many surgical units.

The Ambu ${ }^{\circledR}$ aScope 2TM disposable bronchoscope was initially intended for difficult intubation by anesthetics. This has been used for 7 years and evaluated by several studies [4-6]. Recently, case report showed successful exploration and clearance of common bile duct by open procedure [2].

We presented our case series using Ambu® aScope 2TM disposable bronchoscope for CBD exploration. Our results in terms of duct clearance, complication and hospital stay were in line with series we published from different institution using the standard scope 3 . The results were comparable with other series in the literature [7-9].

Our series showed better outcome in terms of duct clearance and hospital stay in comparison with previous series we published from different institute. Our series showed CBD clearance in 20 from 21 patients, while previous series we published showed 7 of 9 CBD clearances.

The initial average cost of re-usable choledocoscope about $£ 12000$ in addition to sterilization (which could be $£ 200-£ 300$ per choledocoscope), maintenance and other extra costs (storage and cleaning). Disposable bronchoscope costs between $£ 180$ to $£ 220$. We found the advantage of using Ambu ${ }^{\circledR}$ aScope 2TM disposable bronchoscope are cost effectiveness, sterility and free high resolution 6.5" LCD screen provided by the company. We also found that the kit is easy to use, lightweight and good quality image comparable to the image from re-usable choledocoscope. Availability of Ambu ${ }^{\circledR}$ aScope $2 \mathrm{TM}$ disposable bronchoscope makes it convenient and accessible especially if CBD exploration performed in more than one site in the same organisation.

In conclusion, further evaluation is needed to assess the role of disposable bronchoscope in common bile duct exploration. We recommended that using Ambu® aScope 2TM disposable bronchoscope is safe, feasible and cost effective. The comparable results and significant financial effectiveness makes disposable bronchoscope safe alternative to expensive re-usable choledocoscope and it can be applied widely for common bile duct exploration.

Conflict of interest: Mr Yousif Aawsaj, Dr Ibrahim Khalil Ibrahim, Mr Andrew Mitchell and Mr Andrew Gilliam have no conflicts of interest or financial ties to disclose.

\section{REFERENCES}

[1] BV. Dasari, CJ. Tan, KS. Gurusamy, DJ. Martin, G. Kirk, L. McKie, et al., (2013) "Surgical versus endoscopic treatment of bile duct stones," Cochrane Database Syst Rev, vol. 12, pp. 12, 2013

[2] W. Irvine, S. Bradley, E. Minford, "Novel use of Ambu ${ }^{\circledR}$ aScope ${ }^{\mathrm{TM}} 2$ for choledochoscopy," International Journal of Surgery, vol. 12, pp. S21, 2014.

[3] Y. Aawsaj, D. Light, J. Brown, L. Horgan, "Use of the Ambu(®) aScope 2(TM) in laparoscopic common bile duct exploration," Surg endosc, vol. 30, pp. 5153-5155, 2016.

[4] JK. Chan, I. Ng, JP. Ang, SM. Koh, K. Lee, P. Mezzavia, et al. "Randomised controlled trial comparing the Ambu ${ }^{\circledR}$ aScope ${ }^{\mathrm{TM}} 2$ with a conventional fibreoptic bronchoscope in orotracheal intubation of anaesthetised adult patients," Anaesth Intensive Care, vol. 43, pp. 479-84, 2015

[5] I. Hodzovic, AR. Wilkes, "Clinical evaluation of the Ambu ${ }^{\circledR}$ aScope ${ }^{\mathrm{TM}}$," Anaesthesia, vol. 68, pp. 784-5, 2013. 
[6] MS. Kristensen, BB. Fredensborg, "The disposable Ambu aScope vs. a conventional flexible videoscope for awake intubation -- a randomised study," Anaesthesiol Scand, vol. 57, pp. 888-95, 2013.

[7] Y1. Aawsaj, D. Light, L. Horgan, "Laparoscopic common bile duct exploration: 15-year experience in a district general hospital," Surg Endosc, vol. 30, pp. 2563-6, 2016.

[8] DS. Chan, PA. Jain, A. Khalifa, R. Hughes, AL. Baker, "Laparoscopic common bile duct exploration," Br J Surg, vol. 101, pp. 1448-52, 2014.

[9] A. Mattila, J. Luhtala, J. Mrena, H. Kautiainen, I. Kellokumpu, "An audit of short- and long-term outcomes after laparoscopic removal of common bile duct stones in Finland," Surg Endosc, vol. 28, pp. 3451-54, 2014. 